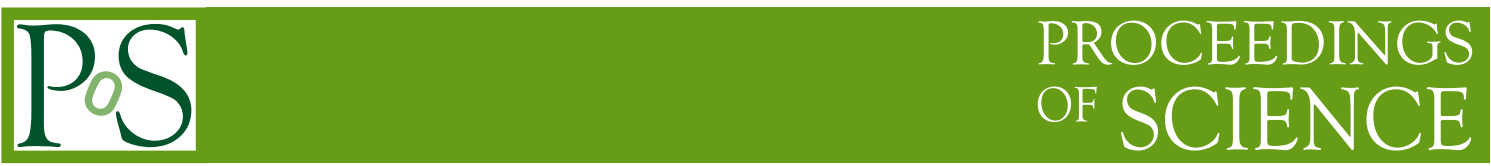

\title{
Hyperon production in annihilation reactions
}

Tord Johansson*广

Dept. of Physics and Astronomy, Uppsala University

E-mail: tord.johansson@physics.uu.se

Strange hyperons are ideal probes of the strong interaction in the transition region between the non-perturbative and perturbative QCD regimes. Prospects for antihyperon-hyperon studies in elementary electron-positron and proton-antiproton annihilation reactions are reviewed with focus on spin degrees of freedom. Special emphasis is put on coming results from BESIII and prospects for the upcoming PANDA experiment at FAIR.

XVII International Conference on Hadron Spectroscopy and Structure - Hadron2017 25-29 September, 2017

University of Salamanca, Salamanca, Spain

\footnotetext{
* Speaker.

${ }^{\dagger}$ Representing the BESIII and PANDA Collaborations.
} 


\section{Introduction}

The understanding of the strong interaction in the transition region between the non-perturbative and perturbative QCD regimes is one of the great challenges of Hadron Physics. Studying systems with strangeness probes the strong interaction in this region at an energy scale of $\approx 100 \mathrm{MeV}$, given by the strange quark mass. This energy scale is of the same magnitude as the $\Lambda_{Q C D}$ of approx. $200 \mathrm{MeV}$ and therefore probes the QCD confinement region. One fundamental question to be posed here is how the properties of baryons consisting of light quarks are affected by replacing light quarks by strange quarks. Baryons with strangeness, i.e. hyperons, have the additional experimental advantage that their ground state configurations mainly decay via the parity violating weak interaction. This makes them a polarimeter for spin observables. Producing hyperon-antihyperon pairs in two-body processes has, in addition, the advantage that spin correlations become accessible. This contribution presents coming results from the BESIII experiment [1] in this direction together with prospects for the future PANDA experiment experiment at FAIR [2].

\section{Hyperon spin observables}

The outlined formalism is valid for any spin $=1 / 2$ hyperon decaying weakly into a baryonmeson pair, but we use the $\Lambda$ hyperon as an example. For an unpolarised initial state in electronpositron or proton-antiproton annihilation into a $\bar{\Lambda} \Lambda$-pair we define a coordinate system in the helicity frame according to Fig.1 below.

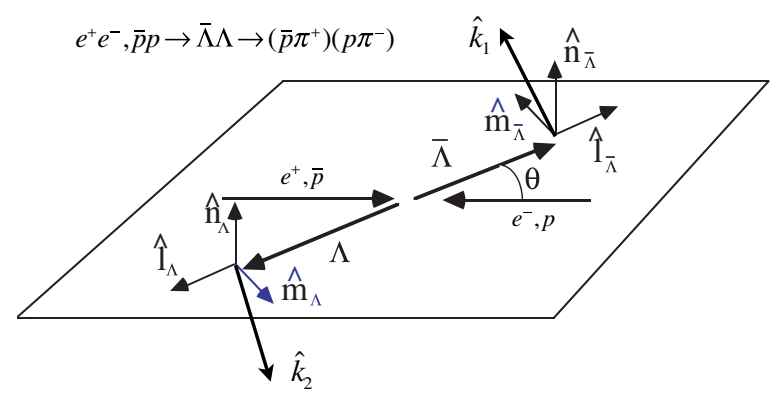

Figure 1: Coordinate system used for the $e^{+} e^{-}, \bar{p} p \rightarrow \bar{\Lambda} \Lambda$ reaction.

There are five (six) spin observables accessible from these reactions: the vector polarisation $P_{n}$ and four (five) spin correlations $C_{n n}, C_{m m}, C_{l l}$ and $C_{m l}$ (charge conjugation imposes $C_{l m}=C_{m l}$ ) and they are defined in Eq. 2.1 below,

$$
I_{\bar{p} p}\left(\theta, \hat{\mathbf{k}}_{1}, \hat{\mathbf{k}}_{2}\right)=\frac{I_{0}^{\bar{\Lambda} \Lambda}}{64}\left(\begin{array}{ll}
1 & \\
+P_{n} & \left(\bar{\alpha}_{\bar{\Lambda}} k_{1 n}+\alpha_{\Lambda} k_{2 n}\right) \\
+C_{n n} & \bar{\alpha}_{\bar{\Lambda}} \alpha_{\Lambda} k_{1 n} k_{2 n} \\
+C_{m m} & \bar{\alpha}_{\bar{\Lambda}} \alpha_{\Lambda} k_{1 m} k_{2 m} \\
+C_{l l} & \bar{\alpha}_{\bar{\Lambda}} \alpha_{\Lambda} k_{l} k_{2 l} \\
+C_{m l} & \bar{\alpha}_{\bar{\Lambda}} \alpha_{\Lambda}\left(k_{1 m} k_{2 l}+k_{1 l} k_{2 m}\right)
\end{array}\right)
$$


where $I_{\bar{p} p}(\theta)$ and $I_{0}^{\bar{\Lambda} \Lambda}$ are the differential and cross sections, respectively, $\hat{\mathbf{k}}_{1}, \hat{\mathbf{k}}_{2}$ are directional cosine vectors of the decay antiprotons/protons in the $\bar{\Lambda} / \Lambda$ rest systems and $\bar{\alpha} / \alpha_{\bar{\Lambda}}$ are the corresponding decay asymmetry parameters. It should be noted that invariance under $\mathrm{CP}$ transformation imposes $\alpha_{\Lambda}=-\bar{\alpha}_{\bar{\Lambda}}$ which makes the $\bar{p} p \rightarrow \bar{\Lambda} \Lambda$ reaction very attractive to search for CP violation in the baryon sector.

\subsection{Hyperon electromagnetic form factors at BESIII}

Electromagnetic Form Factors (EMFF) of baryons are among the mot basic quantities containing information about their internal structure since they provide access to their charge $\left(G_{E}\right)$ and magnetization $\left(G_{M}\right)$ distributions (see Refs. [3, 4, 5, 6] for more details). The most abundant information on EMFF's comes from elastic electron scattering on protons which probe the spacelike (SL) region where the form factors are real (negative four-momentum transfer squared). For nucleons, the spacelike region is well explored, but since hyperons are unstable, elastic scattering measurements are generally unfeasible. Instead, hyperons can be accessed in the time-like (TL) region through the $e^{+} e^{-}$annihilation processes. The low cross section for the $e^{+} e^{-}$processes generally gives small data samples, and most measurements so far, except some $e^{+} e^{-} \rightarrow p \bar{p}$ studies, have only managed to extract the total cross section. The modulus of the ratio of the TL electric magnetic form factors can be extracted from the experimental differential angular distribution according to Eq. 2.2 as given by the one-photon exchange model.

$$
\frac{d \sigma}{d \cos \theta}=\frac{\alpha^{2} \beta}{4 q^{2}}\left(\left|G_{M}\right|^{2}\left(1+\cos ^{2} \theta\right)+\frac{1}{\tau}\left(\mid G_{E}\right]^{2} \sin ^{2}\right) ; \tau=\frac{q^{2}}{4 m_{\Lambda}^{2}}, \beta=\sqrt{1-1 / \tau}
$$

For hyperons however, more information can be gained: the straight-forward access to spin degrees of freedom i.e. $P_{n}$ and the $C_{l m}$, outlined in the previous section, enables extraction of the relative phase $\Delta \Phi$ between $G_{E}$ and $G_{M}$ following Eq.'s 2.3 and 2.4 [8]. This is because in the time-like region, the EMFF's can be complex numbers.

$$
\begin{gathered}
P_{n}=-\frac{\sin 2 \theta \operatorname{Im}\left[G_{E} G_{M}^{*}\right] / \sqrt{\tau}}{\left.\left(\mid G_{E}\right]^{2} \sin 2 \theta\right) / \tau+\left[\left.G_{M}\right|^{2}\left(1+\cos ^{2} \theta\right)\right.}=-\frac{\sin 2 \theta \sin \Delta \phi / \tau}{R \sin 2 \theta / \tau+\left(1+\cos ^{2} \theta\right) / R} ; R=\frac{\left|G_{E}\right|}{\left|G_{M}\right|} \\
C_{l m}=-\frac{\sin 2 \theta \operatorname{Re}\left[G_{E} G_{M}^{*}\right] / \sqrt{\tau}}{\left.\left(\mid G_{E}\right]^{2} \sin 2 \theta\right) / \tau+\left[\left.G_{M}\right|^{2}\left(1+\cos ^{2} \theta\right)\right.}=-\frac{\sin 2 \theta \cos \Delta \phi / \tau}{R \sin 2 \theta / \tau+\left(1+\cos ^{2} \theta\right) / R}
\end{gathered}
$$

A measurement of the polarisation will determine the modulus of the relative phase between $G_{E}$ and $G_{M}$ as given by Eq. 2.3, for which $R$ is determined from the differential cross section. The sign of the relative phase is determined from Eq. 2.4. In fact, it has been shown that an exclusive measurement, as done by BESIII, allows for an unbinned maximum likelihood fit to the data that determine all parameters simultaneously [9]. The BESIII experiment has collected data on strange hyperon pairs at several energies which is presently under analysis. At a centre-of-mass energy of $2.396 \mathrm{GeV}$, a particularly large data sample has been collected with the purpose of measuring a baryon EMFF's phase for the first time, using the $e^{+} e^{-} \rightarrow \bar{\Lambda} \Lambda$ reaction. The "Holy Grail" [4] will thereby be revealed.

A comprehensive overview of BESIII results from hadron timelike form factors is given by P. Larin in these proceedings. 


\subsection{Hyperon spin dynamics with PANDA}

Antiproton-proton reactions are an excellent entrance channel for hyperon studies since these strong interaction processes yield cross sections in the microbarn region for the production of single and doubly strange hyperons. Figure 2 shows the available data on the $\bar{p} p \rightarrow \bar{Y} Y$ reaction in the $\bar{p}$ momentum range accessible for the PANDA experiment given by the HESR storage ring at FAIR. The reaction thresholds are indicated by the arrows. The PS185 experiment at LEAR has mapped out the threshold region up to $2 \mathrm{GeV} / \mathrm{c}$ for the $\bar{p} p \rightarrow \bar{\Lambda} \Lambda$ reaction [11], whereas data are very scarce above $2 \mathrm{GeV} / \mathrm{c}$ [12]. It should be notes that the $\Xi$ cross sections represent a handful events from bubble chamber experiments [12].
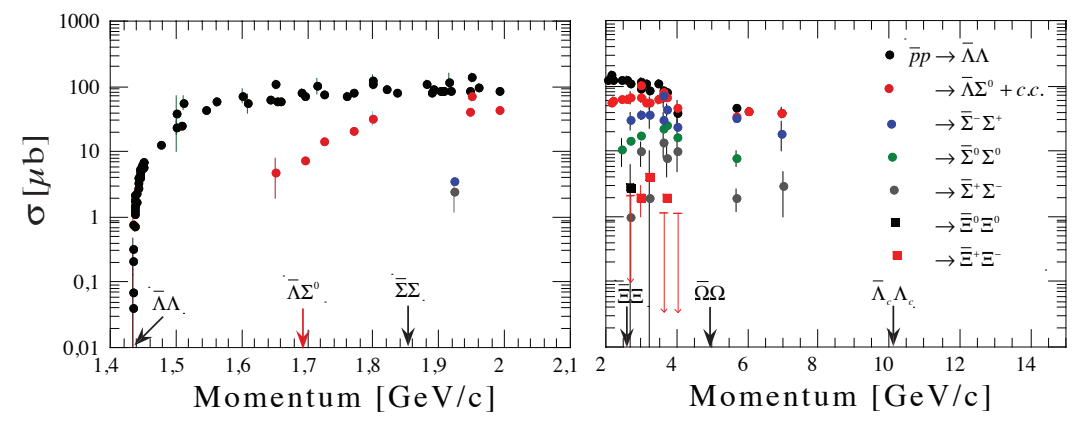

Figure 2: Total cross sections for the $\bar{p} p \rightarrow \bar{Y} Y$ reaction as function of $\bar{p}$ momentum [11, 12].

The PANDA experiment will collect orders of magnitudes larger data samples for these reaction channel that will allow for precision studies of the spin degrees of freedom involved in strangeness and single charm production. Furthermore, spin observables are terra incognita for the $\bar{\Sigma} \Sigma, \bar{\Xi} \Xi, \bar{\Omega} \Omega$ and $\bar{\Lambda}_{c} \Lambda_{c}$ reaction channels. Additional observables become available when studying sequential weak decays. As an example, a measurement of the $\Xi^{-} \rightarrow \Lambda \pi^{-} \rightarrow\left(p \pi^{-}\right) \pi^{-}$decay chain involves two subsequent parity violating weak decays and will give access to the $\beta$ and $\gamma$ decay asymmetry parameters of the $\Xi^{-}$decay from the $\Xi^{-}$and $\Lambda$ polarisations [13]. This is of particular interest since it is expected that the $\beta$ asymmetry parameter is a more sensitive parameter to CP violation than $\alpha$ [14].

\section{Hyperon spectroscopy with PANDA}

The PANDA experiment is not only well suited to study ground state strange hyperons. It is a natural complement to these studies to search for an associated emission of photons and mesons together with these reconstructed hyperons to search for excited states. This opens the venue for hyperon spectroscopy studies. The understanding of the spectra is far from satisfactory, e.g. [10]:

- The level ordering of the light baryon spectra is not understood.

- More states are predicted than observed.

- What are the effective degrees of freedom? Three-quark, diquark-quark or baryon-meson configurations? 
More data are badly needed to shed light on these problems and guide theory. The situation is particularly severe for double and triple strange hyperons, i.e. $\Xi^{*}$ 's, for which only three octet partners of the $N^{*}$ 's have been observed and none of the decouplet partners of the $\Delta^{*}$, i.e. $\Xi^{*}$ 's and $\Omega^{*}$ 's [7]. PANDA is well suited for such studies since no extra production kaons are needed in $\bar{p} p$ collisions for strangeness conservation. Furthermore, there is an equal yield of $Y^{*}$ 's and $\bar{Y}^{*}$ 's which allows for consistency checks.

It is expected that the cross section for producing excited hyperon is of the same order of magnitude as the ground state hyperons.

\section{Conclusions}

There are good prospects for hyperon physics in the near future for the BESIII experiment and in the more distant future with PANDA.

\section{References}

[1] M. Ablikim et al., Design and construction of the BESIII detector, Nucl. Instr. Meth. A 614, 345 (2010).

[2] W. Erni et al., Physics Performance Report for PANDA, arXiv:0903.3905 (2009).

[3] C. Perdrisat, V. Punjabi V. and M. Vanderhaegen, Nucleon electromagnetic form factors Prog. Part. Nucl. Phys. 59, 694 (2007).

[4] A.Denig and V. Salmé, Nucleon electromagnetic form factors in the timelike region Prog. Part. Nucl. Phys. 68, 113 (2013).

[5] V. Punjabi et al., The Structure of the Nucleon: Elastic Electromagnetic Form Factors, Eur. Phys. J A 5179 (2015) [nucl-ex/1503.01452v4].

[6] S. Pacetti, R. Baldini Ferrolli and E. Tomasi-Gustafsson, Proton electromagnetic form factors: Basic notions, present achievements and future perspectives. Phys. Rep.550-551, 1 (2015).

[7] C. Patrignani et.al., The Review of Particle Physics (2017), Chin. Phys. C, 40 , 100001 (2016) and 2017 update.

[8] G. Fäldt, Polarization variables in the $e^{+} e^{-} \rightarrow \bar{\Lambda} \Lambda$ reaction, Eur. Phys. J. A52 no.5 141 (2016).

[9] G. Fäldt and A. Kupsc, Hadronic structure functions in the $e^{+} e^{-} \rightarrow \bar{\Lambda} \Lambda$ reaction, Phys. Lett. B772 16 (2017).

[10] E. Klempt E. and J.-M. Richard, Baryon spectroscopy, Rev. Mod. Phys. 821095 (2010).

[11] T. Johansson, Antihyperon-Hyperon Production in Antiproton-Proton Collisions, Proc. Eight Int. Conf. on Low Energy Antiproton Physics (LEAP’05), AIP Conf. Proc. 79695 (2004).

[12] V. Flamino et.al., COMPLIATION OF CROSS-SECTIONS III: $p$ and $\bar{p}$ INDUCED REACTIONS, CERN-HERA 84-01.

[13] T.D. Lee and C.D. Yang, General Partial Wave Analysis of the Decay of a Hyperon of Spin 1/2, Phys. Rev. 1081645 (1957)

[14] J.F. Donoghue, X.G. He and S. Pakavasa, Hyperon decays and CP violation, Phys. Rev. D34 833 (1986). 